\title{
Foraging Patterns of Birds in Resource Partitioning in Tropical Mixed Dry Deciduous Forest, India
}

\author{
Nirmala Thivyanathan \\ Principal, Research Centre of Zoology, Jayaraj Annapackiam College for Women, Periyakulam, Theni District, Tamilnadu, India
}

\section{Email address:}

principal@annejac.com

\section{To cite this article:}

Nirmala Thivyanathan. Foraging Patterns of Birds in Resource Partitioning in Tropical Mixed Dry Deciduous Forest, India. Journal of Energy and Natural Resources. Vol. 5, No. 2, 2016, pp. 16-29. doi: 10.11648/j.jenr.20160502.11

Received: February 18, 2016; Accepted: May 21, 2016; Published: May 23, 2016

\begin{abstract}
A study was undertaken in the tropical mixed dry deciduous forest of India. Direct observation on foraging of birds was made on twelve days in a month within four hours after sunrise with direct observation. For each foraging attempt microhabitat details such as the foraging height, substrate, method, canopy and the plant species were recorded. Vegetation profile consisted of tree species from 2 to $6 \mathrm{~m}$ and shrubs from 0 to $1 \mathrm{~m}$ height. In total, 3982 foraging observations were made on 36 bird species. A higher percentage of foraging manoeuvre was recorded at 3-6m height. 29 bird species were gleaner. Majority of the canopy layers used for foraging of bird species were edge edge (23\%) followed by ground (18\%) and middle lower (17\%). Grey Jungle Fowl, Vernal Hanging Parrot and Red-rumped Swallow are specialists. The higest mean niche overlap among the species was found in method followed by canopy and height. The two major guilds are gleaner and sallier.
\end{abstract}

Keywords: Foraging Method, Foraging Substrate, Foraging Canopy, Foraging Height, Guild, Niche Overlap, Resource Partitioning, Tropical Mixed Dry Deciduous Forest

\section{Introduction}

Birds prefer some specific habitats and coexist as guilds with the available pattern of food resources [1]. Guild segregates themselves into specific ecological niches by adopting foraging behaviour and differs in microhabitat use and foraging tactics [2]. The foraging tactics include various methods to exploit the resources. Insectivore birds exhibit different methods of exploiting resources such as gleaning, sallying, probing, pouncing and hawking [3], [4], [5].

Although resource partitioning has been well documented for bird species from temperate forests [3], [6], [7], no such studies are available in India except the study of Gokula and Vijayan [5] in the dry deciduous forest of Mudumalai Wildlife Sanctuary. Moreover, knowledge of the ways in which birds exploit resources within a forest will increase the understanding of their habitat use and the essential requirements for their survival. The following objectives were set to analyse the patterns of feeding behavior, method of feeding and microhabitat use by birds in the mixed dry deciduous forest.

\section{Study Area}

The study was undertaken in the tropical mixed dry deciduous forest of Anaikatty hills [8], the foothills of the Nilgiri in the Nilgiri Biosphere Reserve, Western Ghats, India situated at an elevation of about $610-1200 \mathrm{~m}$ above MSL between $76^{\circ} 39^{\prime}$ and $76^{\circ} 47^{\prime} \mathrm{E}$ and from $11^{\circ} 5^{\prime}$ to $11^{\circ}$ $31^{\prime} \mathrm{N}$ in Coimbatore, TamilNadu, Southern India. The climate is moderate and pleasant for most part of the year except summer which is relatively hot and dry.

Based on the climate, four different seasons were observed as follows. Southwest monsoon (June, July and August): The study area received $5 \%$ of the total annual rainfall during this season. The mean rainfall received was around $40 \mathrm{~mm}$. Northeast monsoon (September, October and November): The study area received more than half $(69 \%)$ of the total annual rainfall during this season. The mean rainfall received was around $500 \mathrm{~mm}$.

Winter (December, January and February): It was the least rainy period of the year with the annual rainfall of $34 \mathrm{~mm}$. This season was the colder period with the minimum 
temperature falling to $18^{\circ} \mathrm{C}$. Summer (March, April and May): This area received $21 \%$ of the annual rainfall in this season from the pre-monsoon showers. This was the period of maximum temperature, which leaped up to $37^{\circ} \mathrm{C}$ with low relative humidity.

Temperature varied between $18^{\circ} \mathrm{C}$ and $37^{\circ} \mathrm{C}$ and Relative humidity showed fluctuation in different seasons between $31 \%-75 \%$ at $08: 30 \mathrm{hrs}$. and $72 \%-89 \%$ at $17: 30 \mathrm{hrs}$. Monthly windspeed varied between 3 and $14 \mathrm{~km} / \mathrm{h}$. The tropical mixed dry deciduous forest, India has the major tree community of Acacia leucophloea, Ziziphus mauritiana, Chloroxylon swietenia, Albizia amara, Tamarindus indicus, Albizia lebbeck, Acacia polyacantha, Diospyros ferrea, Cassia fistula and Commiphora caudata. Major shrubs are Chromolaena odorata, Elaeodendron glaucum, Pavetta indica, Lantana camara, Randia dumetorum, Premna tomentosa, Flacourtia indica and Mundulea sericea.

\section{Materials and Methods}

Foraging records of birds were made during May 1999 to May 2001 on twelve days in a month from the tropical mixed dry deciduous forest, India. Most of the observations were done within four hours after sunrise. This is the most active foraging time for birds [9]. Only initial record was taken from any individual encountered as done by MacNally [7] to provide precise estimate of foraging location rather than that of the subsequent ones [10].

Table 1. Definition of foraging activities used to assess guild structure of avifauna.

\begin{tabular}{ll}
\hline Foraging method & Sub categories \\
\hline \multirow{4}{*}{ Sally } & Above canopy-sally \\
& Below canopy-sally \\
& Herb-sally \\
& Shrub-sally \\
& Sally (sally to the ground) \\
& Flower-glean \\
& Fruit-gleaning \\
& Ground-gleaning \\
Glean & Litter-gleaning \\
& Main trunk-gleaning \\
& Secondary branch-gleaning \\
& Twig-glean \\
Pounce & Leaf-glean \\
& Ground-pounce \\
Probing & Ground-probing \\
& Litter-probing \\
Tear & Main trunk-probing \\
Hover & Secondary branch-probing \\
\hline
\end{tabular}

For each foraging attempt microhabitat details such as the foraging height, substrate, method, canopy and the plant species at which the prey was found were recorded. Foraging attempts were assigned to 12 height categories. A substrate is the place from where food is taken by birds in 7 different areas. Foraging methods of birds were categorized as, Glean, Probe, Sally or fly catching and Pounce, To cluster the species on a micro level, these methods were classified further into finer levels based on the substrate, which is given in Table 1 and described by Crome [3] and expanded by Holmes et al., [4], Ramsen and Robinson [11] and MacNally [12].

The canopy layers used by the bird species were classified into ten layers and were possibly distinguished from three layers namely lower canopy, middle canopy and upper/edge canopy (Figure 1). Lower canopy was further distinguished as lower center, lower middle and lower edge. Middle canopy was classified further into middle center, middle middle and middle edge. c). Upper/edge canopy was classified as edge center, edge middle and edge edge. d). Birds, which do not use plant at all for its prey was grouped under ground/air/under canopy.

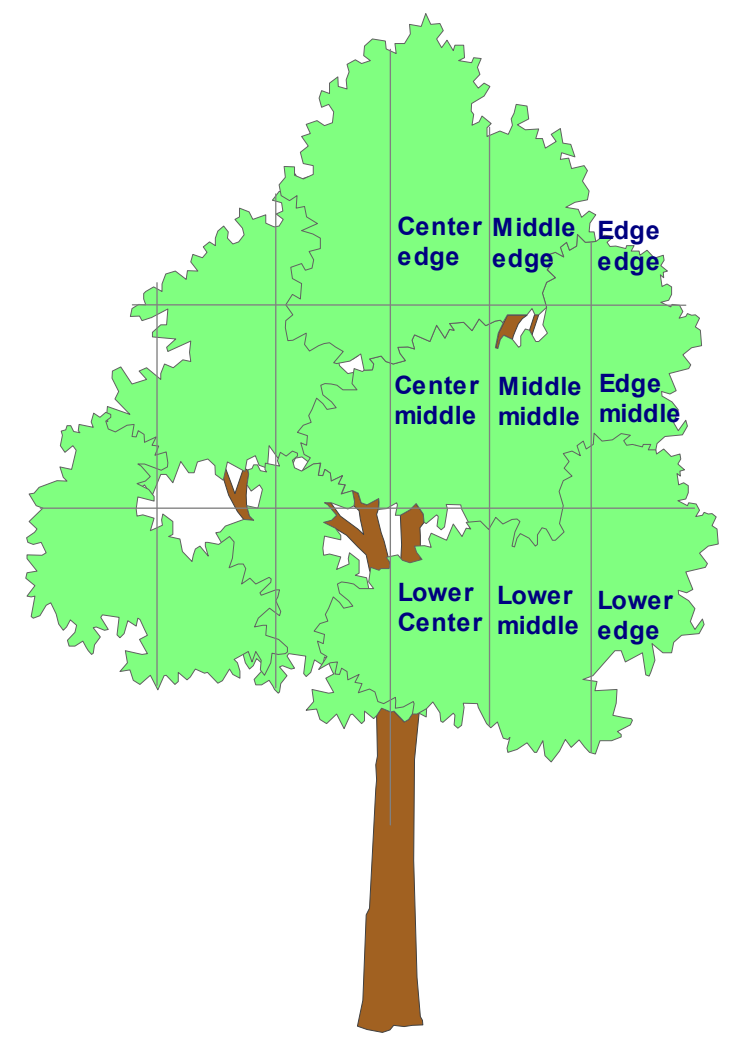

Figure 1. Diagrammatic Representation of the Canopy Layers of a Plant.

As thirty independent observations are recommended to represent the behavior of a bird accurately [13], species with more than 30 observations were taken for analysis.

\section{Statistical Analysis}

\subsection{Specialist-Index $J^{\prime}$}

The foraging specialization of each foraging parameter (method, substrate, height and canopy) was analyzed using the Shannon-Weaver [14] index. These values were then converted to a standardized range using the formula $\mathrm{J}^{\prime}=$ $H^{\prime} / H_{\max }$ (Where $\mathrm{J}^{\prime}=$ specialization and $H_{\max }=$ the maximum $H^{\prime}$ 'value) following Crome [3] and Recher et al. [6]. $J$ value ranges between one and zero, with foraging specialization increases as $J^{\prime}$ decreases. 


\subsection{Niche Overlap}

The extent to which resource use overlaps between species pairs is niche overlap. The degree of species overlap in niche utilization for the different categories recorded (foraging method, substrate, canopy and foraging height) has been quantitatively expressed using Horn's index [15].

\subsection{Cluster Analysis}

To compare foraging behavior (substrate, height, canopy use and method adopted) by various species, cluster analyses were performed on a data matrix (species * characteristics), following Holmes et al. [4]. This analysis used the unweighted pair group clustering method with arithmetic averages (UPGMA) and Squared Euclidean Distance [16], [17]. The SPSS statistical software [18] was used for the data analyses.

\section{Results}

In total, 3982 foraging observations were made on 36 species in the tropical mixed dry deciduous forest, India (Table 2).

Table 2. Number of foraging records on each bird species observed in the tropical mixed dry deciduous forest during $1999-2001$.

\begin{tabular}{|c|c|c|c|c|}
\hline S. No & Common name of the species & Scientific name & Family & Number of foraging observations \\
\hline 1 & GREY JUNGLEFOWL & Gallus sonneratii & Phasianidae & 94 \\
\hline 2 & INDIAN PEAFOWL & Pavo cristatus & Phasianidae & 36 \\
\hline 3 & BLOSSOM-HEADED PARAKEET & Psittacula roseate & Psittacidae & 45 \\
\hline 4 & MALABAR PARAKEET & Psittacula columboides & Psittacidae & 225 \\
\hline 5 & VERNAL HANGING PARROT & Loriculus vernalis & Psittacidae & 53 \\
\hline 6 & BLUE-FACED MALKOHA & Phaenicophaeus viridirostris & Cuculidae & 74 \\
\hline 7 & GREEN BEE-EATER & Merops orientalis & Meropidae & 42 \\
\hline 9 & COMMON HOOPOE & Upupa epops & Upupidae & 35 \\
\hline 10 & BROWN-HEADED BARBET & Megalaima zylanica & Capitonidae & 31 \\
\hline 11 & STREAK-THROATED WOODPECKER & Picus xanthopygaeus & Picidae & 28 \\
\hline 12 & PALE-BILLED FLOWERPECKER & Dicaeum erythrorynchos & Picidae & 124 \\
\hline 13 & RED-RUMPED SWALLOW & Hirundo daurica & Hirundinidae & 49 \\
\hline 14 & RED-WHISKERED BULBUL & Pycnonotus jocosus & Pycnonotidae & 84 \\
\hline 15 & RED-VENTED BULBUL & Pycnonotus cafer & Pycnonotidae & 102 \\
\hline 16 & WHITE-BROWED BULBUL & Pycnonotus luteolus & Pycnonotidae & 333 \\
\hline 18 & COMMON IORA & Aegithina typhia & Irenidae & 368 \\
\hline 19 & BLUE-WINGED LEAFBIRD & Chloropsis cochinchinensis & Irenidae & 115 \\
\hline 20 & TAWNY-BELLIED BABBLER & Dumetia hyperythra & Muscicapidae & 125 \\
\hline 21 & JUNGLE BABBLER & Turdoides striatus & Muscicapidae & 240 \\
\hline 22 & YELLOW-BILLED BABBLER & Turdoides affinis & Muscicapidae & 189 \\
\hline 23 & BLYTH'S REED WARBLER & Phylloscopus reguloides & Muscicapidae & 128 \\
\hline 24 & COMMON TAILORBIRD & Orthotomus sutorius & Muscicapidae & 40 \\
\hline 25 & GREENISH WARBLER & Phylloscopus trochiloides & Muscicapidae & 159 \\
\hline 26 & LARGE-BILLED LEAF WARBLER & Phylloscopus magnirostris & Muscicapidae & 147 \\
\hline 27 & ASIAN PARADISE FLYCATCHER & Terpsiphone paradise & Muscicapidae & 82 \\
\hline 28 & PLAIN FLOWERPECKER & Dicaeum concolor & Dicaeidae & 74 \\
\hline 29 & PURPLE-RUMPED SUNBIRD & Nectarinia zeylonica & Nectariniidae & 361 \\
\hline 30 & LOTEN'S SUNBIRD & Nectarinia lotenia & Nectariniidae & 90 \\
\hline 31 & PURPLE SUNBIRD & Nectarinia asiatica & Nectariniidae & 57 \\
\hline 32 & JUNGLE MYNA & Acridotheres fuscus & Sturnidae & 72 \\
\hline 35 & ASHY DRONGO & Dicrurus leucophaeus & Dicruridae & 33 \\
\hline \multirow[t]{2}{*}{36} & WHITE-BELLIED DRONGO & Dicrurus caerulescens & Dicruridae & 103 \\
\hline & Total & & & 3982 \\
\hline
\end{tabular}

Nomenclature following Grimmette et al. (1998)

\subsection{Foraging Height}

All the 12 height categories were utilized by 36 bird species in the tropical mixed dry deciduous forest, India (Table 3). Although most species fed over a broad range of heights, they were grouped according to the layer of vegetation in which the majority of their foraging was recorded. Foliage was partitioned as three layers of strata; ground $(0 \mathrm{~m})$, shrub/short trees $(0.1-3)$, and tree layers $(>3)$.
In the community as a whole, a higher percentage of foraging manoeuvre were recorded in the layers of 3-6m height.

Six species foraged mainly at ground level. Among them, Grey Junglefowl absolutely used the ground layer while Jungle Myna, Yellow-billed Babbler, Indian Peafowl, Common Hoopoe, and Jungle Babbler showed variety in their height preference.

The $0.1-3 \mathrm{~m}$ height category of shrub and short tree layers 
were utilized by Blossom-headed Parakeet, Tawny-bellied Babbler, Loten's Sunbird, Common Tailorbird, Red-vented Bulbul, White-browed Bulbul, Blyth's Reed Warbler, Purplerumped Sunbird and Asian Paradise Flycatcher.

The tree layer $(>3 \mathrm{~m})$ was used by 21 bird species. Within the tree layers, higher percentage of foraging manoeuvre was recorded in the 3-6m height category. All the foraging attacks of the Ashy Drongo, Large-billed Leaf Warbler, Black Drongo, Vernal Hanging Parrot and Red-rumped Swallow were at $>6 \mathrm{~m}$ height. For the foraging community as a whole in the tropical mixed dry deciduous forest, a higher number of foraging manoeuvres was recorded in the tree layers $(>3 \mathrm{~m}$ height).

\subsection{Foraging Substrate}

Majority of the bird species used foliage (24 bird species) followed by twigs ( 22 bird species) as their substrate (Table 4). Only 11 species used ground and flower to find their food.
The ground-foraging guild was with five species viz. Jungle Babbler, Grey Jungle fowl, Indian Peafowl, Common Hoopoe and Yellow-billed Babbler.

Streak-throated Woodpecker and Black-Hooded Oriole largely obtained their prey from the trunk. In addition to this substrate, these birds also used twigs and fruits. Five bird species such as Blyth's Reed Warbler, Bluewinged Leafbird, Plain Flowerpecker, Black Bulbul and Large-billed Leaf Warbler used this substrate. Blue-faced Malkoha, Greenish Warbler, Tawny-bellied Babbler, Common Tailorbird and Common Iora used exclusively twigs as substrate (Table 4). Only Vernal Hanging Parrot alone used flower as its substrate while Parakeets used fruit predominantly with a little overlap of flower. Other species such as Bulbuls and Large Green Barbet used this substrate and also other substrates for their prey. Red-rumped Swallow obtained its prey exclusively from air. Drongos predominantly used air for their prey and in addition, they also used foliage to a lesser extent.

Table 3. Percentage of prey attacks by different species of birds at various height categories in the tropical mixed dry deciduous forest, India.

\begin{tabular}{|c|c|c|c|c|c|c|c|c|c|c|c|c|c|}
\hline \multirow{2}{*}{ Name of the bird species } & \multicolumn{13}{|c|}{ Foraging heights (m) } \\
\hline & 0 & $0.1-1$ & $1-2$ & $2-3$ & $3-4$ & $4-5$ & $5-6$ & $6-7$ & $7-8$ & $8-9$ & $9-10$ & $>10$ & $\mathbf{H}^{\prime}$ \\
\hline Grey junglefowl & 100 & 0 & 0 & 0 & 0 & 0 & 0 & 0 & 0 & 0 & 0 & 0 & 0.0 \\
\hline Common hoopoe & 91 & 0 & 0 & 3 & 0 & 0 & 3 & 3 & 0 & 0 & 0 & 0 & 1.9 \\
\hline Yellow-billed babbler & 97 & 2 & 0 & 0 & 0 & 0 & 1 & 0 & 0 & 0 & 0 & 0 & 0.4 \\
\hline Jungle babbler & 81 & 6 & 4 & 4 & 1 & 1 & 2 & 0 & 0 & 0 & 0 & 0 & 0.7 \\
\hline Indian peafowl & 77 & 23 & 0 & 0 & 0 & 0 & 0 & 0 & 0 & 0 & 0 & 0 & 1.5 \\
\hline Blossom-headed parakeet & 0 & 88 & 0 & 0 & 0 & 0 & 0 & 0 & 0 & 0 & 2 & 9 & 0.3 \\
\hline Tawny-bellied babbler & 0 & 72 & 24 & 4 & 0 & 0 & 0 & 0 & 0 & 0 & 0 & 0 & 0.7 \\
\hline Loten's sunbird & 0 & 44 & 11 & 2 & 8 & 19 & 8 & 6 & 2 & 0 & 0 & 0 & 1.7 \\
\hline Common tailorbird & 0 & 48 & 30 & 10 & 8 & 5 & 0 & 0 & 0 & 0 & 0 & 0 & 0.9 \\
\hline Red-vented bulbul & 0 & 40 & 15 & 6 & 14 & 1 & 4 & 5 & 1 & 3 & 0 & 12 & 1.8 \\
\hline White-browed bulbul & 2 & 16 & 30 & 22 & 17 & 12 & 1 & 1 & 1 & 0 & 0 & 0 & 0.9 \\
\hline Blyth's reed warbler & 0 & 27 & 30 & 18 & 15 & 8 & 2 & 0 & 0 & 0 & 1 & 0 & 1.5 \\
\hline Asian paradise flycatcher & 0 & 18 & 26 & 45 & 5 & 5 & 1 & 0 & 0 & 0 & 0 & 0 & 1.4 \\
\hline Brown-headed barbet & 0 & 0 & 6 & 29 & 10 & 6 & 19 & 16 & 10 & 0 & 0 & 3 & 2.3 \\
\hline Black bulbul & 0 & 0 & 0 & 26 & 0 & 3 & 38 & 24 & 0 & 0 & 9 & 0 & 1.5 \\
\hline Red-whiskered bulbul & 0 & 14 & 19 & 24 & 8 & 12 & 12 & 7 & 1 & 1 & 1 & 0 & 2.2 \\
\hline Streak-throated woodpecker & 0 & 0 & 7 & 21 & 57 & 0 & 11 & 0 & 4 & 0 & 0 & 0 & 0.3 \\
\hline Green bee-eater & 0 & 19 & 0 & 0 & 52 & 7 & 0 & 0 & 0 & 0 & 0 & 21 & 0.7 \\
\hline Malabar parakeet & 0 & 3 & 2 & 3 & 32 & 28 & 12 & 4 & 7 & 3 & 4 & 3 & 1.2 \\
\hline Blue-winged leafbird & 0 & 0 & 0 & 5 & 30 & 39 & 17 & 5 & 2 & 1 & 1 & 0 & 1.4 \\
\hline Purple sunbird & 0 & 5 & 23 & 2 & 19 & 7 & 28 & 0 & 11 & 0 & 5 & 0 & 2.2 \\
\hline Pale-billed flowerpecker & 0 & 4 & 9 & 10 & 27 & 16 & 21 & 4 & 3 & 3 & 3 & 1 & 1.9 \\
\hline Common iora & 0 & 3 & 13 & 17 & 22 & 17 & 15 & 7 & 2 & 0 & 2 & 2 & 0.9 \\
\hline Blue-faced malkoha & 19 & 14 & 3 & 5 & 20 & 23 & 8 & 4 & 0 & 3 & 0 & 1 & 2.3 \\
\hline Greenish warbler & 0 & 0 & 1 & 8 & 16 & 25 & 18 & 6 & 16 & 3 & 2 & 7 & 1.5 \\
\hline White-bellied drongo & 4 & 1 & 0 & 1 & 13 & 22 & 14 & 17 & 12 & 10 & 4 & 4 & 2.1 \\
\hline Chestnut-headed bee-eater & 2 & 5 & 7 & 2 & 2 & 16 & 16 & 13 & 13 & 0 & 2 & 23 & 2.7 \\
\hline Ashy drongo & 0 & 0 & 0 & 0 & 3 & 15 & 30 & 15 & 3 & 0 & 18 & 15 & 1.9 \\
\hline Large-billed leaf warbler & 0 & 1 & 6 & 12 & 19 & 28 & 22 & 7 & 3 & 2 & 1 & 0 & 1.5 \\
\hline Black drongo & 8 & 3 & 1 & 3 & 4 & 0 & 21 & 10 & 22 & 3 & 7 & 19 & 2.4 \\
\hline Vernal hanging parrot & 0 & 0 & 0 & 0 & 2 & 0 & 2 & 2 & 34 & 8 & 53 & 0 & 0.9 \\
\hline Red-rumped swallow & 0 & 0 & 0 & 2 & 0 & 29 & 0 & 0 & 0 & 0 & 59 & 10 & 0.6 \\
\hline
\end{tabular}


Table 4. Percentage use of various foraging substrates by different species of birds in the tropical mixed dry deciduous forest, India.

\begin{tabular}{|c|c|c|c|c|c|c|c|}
\hline Name of the bird species & Ground & Trunk & Foliage & Twigs & Flower & Fruit & Air \\
\hline ASHY DRONGO & 0 & 0 & 0 & 0 & 24 & 0 & 76 \\
\hline BLACK BULBUL & 0 & 1 & 45 & 49 & 0 & 5 & 0 \\
\hline BLACK DRONGO & 0 & 0 & 1 & 0 & 0 & 0 & 97 \\
\hline BLACK-HOODED ORIOLE & 0 & 66 & 0 & 32 & 0 & 2 & 0 \\
\hline BLOSSOM-HEADED PARAKEET & 0 & 0 & 0 & 0 & 7 & 93 & 0 \\
\hline BLYTH'S REED WARBLER & 0 & 0 & 56 & 44 & 0 & 0 & 0 \\
\hline MALABAR PARAKEET & 0 & 0 & 0 & 0 & 2 & 98 & 0 \\
\hline BLUE-FACED MALKOHA & 24 & 8 & 9 & 35 & 0 & 23 & 0 \\
\hline GREY JUNGLEFOWL & 97 & 0 & 3 & 0 & 0 & 0 & 0 \\
\hline BLUE-WINGED LEAFBIRD & 0 & 0 & 56 & 17 & 20 & 8 & 0 \\
\hline COMMON HOOPOE & 91 & 6 & 0 & 3 & 0 & 0 & 0 \\
\hline COMMON IORA & 0 & 0 & 27 & 72 & 0 & 0 & 0 \\
\hline JUNGLE BABBLER & 81 & 2 & 0 & 17 & 0 & 0 & 0 \\
\hline JUNGLE MYNA & 39 & 3 & 0 & 0 & 53 & 6 & 0 \\
\hline LARGE-BILLED LEAF WARBLER & 0 & 0 & 90 & 10 & 0 & 0 & 0 \\
\hline BROWN-HEADED BARBET & 0 & 6 & 6 & 0 & 0 & 87 & 0 \\
\hline VERNAL HANGING PARROT & 0 & 0 & 0 & 0 & 100 & 0 & 0 \\
\hline LOTEN'S SUNBIRD & 0 & 0 & 0 & 6 & 93 & 1 & 0 \\
\hline STREAK-THROATED WOODPECKER & 0 & 93 & 0 & 7 & 0 & 0 & 0 \\
\hline PLAIN FLOWERPECKER & 0 & 0 & 46 & 40 & 14 & 0 & 0 \\
\hline INDIAN PEAFOWL & 74 & 0 & 3 & 0 & 0 & 23 & 0 \\
\hline ASIAN PARADISE FLYCATCHER & 0 & 0 & 12 & 0 & 0 & 0 & 88 \\
\hline GREENISH WARBLER & 0 & 9 & 27 & 64 & 0 & 0 & 0 \\
\hline PURPLE-RUMPED SUNBIRD & 0 & 0 & 5 & 7 & 87 & 1 & 0 \\
\hline PURPLE SUNBIRD & 0 & 7 & 16 & 4 & 74 & 0 & 0 \\
\hline TAWNY-BELLIED BABBLER & 0 & 0 & 14 & 85 & 0 & 1 & 0 \\
\hline RED-RUMPED SWALLOW & 0 & 0 & 0 & 0 & 0 & 0 & 100 \\
\hline RED-VENTED BULBUL & 2 & 12 & 4 & 12 & 0 & 71 & 0 \\
\hline RED-WHISKERED BULBUL & 1 & 4 & 1 & 24 & 0 & 70 & 0 \\
\hline GREEN BEE-EATER & 0 & 0 & 21 & 0 & 0 & 0 & 79 \\
\hline COMMON TAILORBIRD & 3 & 0 & 35 & 58 & 0 & 5 & 0 \\
\hline PALE-BILLED FLOWERPECKER & 0 & 0 & 15 & 21 & 64 & 0 & 0 \\
\hline WHITE-BROWED BULBUL & 2 & 0 & 1 & 15 & 0 & 77 & 5 \\
\hline WHITE-BELLIED DRONGO & 0 & 0 & 2 & 0 & 0 & 0 & 98 \\
\hline YELLOW-BILLED BABBLER & 97 & 0 & 1 & 2 & 0 & 0 & 0 \\
\hline CHESTNUT-HEADED BEE-EATER & 0 & 0 & 13 & 0 & 0 & 0 & 88 \\
\hline Substrate preference by Number of Bird Species & 11 & 12 & 24 & 22 & 11 & 16 & 8 \\
\hline
\end{tabular}

Table 5. Percentage of prey attack manoeuvres by different bird species in the tropical mixed dry deciduous forest, India.

\begin{tabular}{llllll}
\hline Name of the Bird Species & Glean & hover & Pounce & Probe & Sally \\
\hline BLACK BULBUL & 100 & 0 & 0 & 0 & 0 \\
BLACK-HOODED ORIOLE & 83 & 0 & 17 & 0 & 0 \\
BLOSSOM-HEADED PARAKEET & 100 & 0 & 0 & 0 & 0 \\
BLYTH'S REED WARBLER & 100 & 0 & 0 & 0 & 0 \\
MALABAR PARAKEET & 100 & 0 & 0 & 0 & 0 \\
BLUE-FACED MALKOHA & 99 & 0 & 0 & 0 \\
GREY JUNGLEFOWL & 95 & 0 & 0 & 5 & 0 \\
BLUE-WINGED LEAFBIRD & 100 & 0 & 0 & 0 & 0 \\
COMMON HOOPOE & 74 & 0 & 0 & 0 & 0 \\
COMMON IORA & 100 & 0 & 0 & 0 & 0 \\
JUNGLE BABBLER & 100 & 0 & 0 & 0 & 0 \\
JUNGLE MYNA & 97 & 0 & 0 & 0 & 0 \\
LARGE-BILLED LEAF WARBLER & 100 & 0 & 0 & 0 & 0 \\
BROWN-HEADED BARBET & 100 & 0 & 0 & 0 & 0 \\
VERNAL HANGING PARROT & 100 & 0 & 0 & 0 & 0 \\
LOTEN'S SUNBIRD & 100 & 0 & 0 & 0 & 0 \\
STREAK-THROATED WOODPECKER & 71 & 0 & 0 & 0 \\
PLAIN FLOWERPECKER & 100 & 0 & 0 & 0 \\
INDIAN PEAFOWL & 67 & 0 & 0 & 0 \\
GREENISH WARBLER & 100 & 0 & 0 & 0 \\
PURPLE-RUMPED SUNBIRD & 100 & 0 & 0 & 0 \\
PURPLE SUNBIRD & 100 & 0 & 0 & 0 \\
TAWNY-BELLIED BABBLER & 100 & 0 & 0 & 0 \\
RED-VENTED BULBUL & 99 & 0 & 1 & 0 \\
RED-WHISKERED BULBUL & 100 & 0 & 0 & 0 \\
\hline
\end{tabular}




\begin{tabular}{|c|c|c|c|c|c|}
\hline Name of the Bird Species & Glean & hover & Pounce & Probe & Sally \\
\hline COMMON TAILORBIRD & 100 & 0 & 0 & 0 & 0 \\
\hline PALE-BILLED FLOWERPECKER & 100 & 0 & 0 & 0 & 0 \\
\hline WHITE-BROWED BULBUL & 95 & 0 & 0 & 0 & 5 \\
\hline YELLOW-BILLED BABBLER & 100 & 0 & 0 & 0 & 0 \\
\hline RED-RUMPED SWALLOW & 0 & 100 & 0 & 0 & 0 \\
\hline ASHY DRONGO & 24 & 0 & 0 & 0 & 76 \\
\hline BLACK DRONGO & 0 & 0 & 0 & 0 & 100 \\
\hline ASIAN PARADISE FLYCATCHER & 0 & 0 & 0 & 0 & 100 \\
\hline GREEN BEE-EATER & 0 & 0 & 0 & 0 & 100 \\
\hline WHITE-BELLIED DRONGO & 0 & 0 & 0 & 0 & 100 \\
\hline CHESTNUT-HEADED BEE-EATER & 0 & 0 & 0 & 0 & 100 \\
\hline
\end{tabular}

\subsection{Foraging Methods}

Birds such as gleaner $(88 \%)$, sallier $(10 \%)$, prober $(1 \%)$, pouncer and hoverer (1\%) were recorded from this forest (Table 5). Twenty-nine species were recorded as gleaner, of which 24 species predominantly used (100\%) gleaning. Six species such as Asian Paradise Flycatcher, White-bellied Drongo, Green Bee-eater, Black Drongo, Ashy Drongo and Chestnut-headed Bee-eater used sallying to obtain their prey. Except Ashy Drongo, all other birds of this guild used sally as the only prey attacking manoeuvre. Red-rumped Swallow was recognized as hoverer or aerial capture, which used this method alone as the prey-attacking manoeuvre.

Prey attack manoeuvre by gleaners: Since gleaning formed the major method adopted by the birds of tropical mixed dry deciduous forest, India, it's usage was further bifurcated into eight types (Table 6). In total, gleaning of flower (21\%), fruit $(21 \%)$ and twig $(21 \%)$ formed $63 \%$ of gleaning. Gleaning on ground (18\%) and leaf (11\%) was comparatively less, while on trunk (4\%) and stem (4\%) it was very little.

Flower Gleaning: Six species exploited the flowers by gleaning for nectar. Vernal Hanging Parrot alone used only this method for feeding. Loten's Sunbird, Purple-rumped Sunbird and Purple Sunbird used this method predominantly while Pale-Billed Flowerpecker and Jungle Myna used this method frequently (Table 6). Fruit Gleaning: Malabar Parakeet, Blossom-headed Parakeet frequently used this method along with flower gleaning. Brown-headed Barbet, White-browed Bulbul, Red-vented Bulbul and Redwhiskered Bulbul also used this method along with other methods. Ground Gleaning: Yellow-billed (White-headed) Babbler, Grey Junglefowl, Jungle Babbler, Indian Peafowl and Common Hoopoe formed the groud gleaner to get their prey from ground and overlap with litter gleaning.

Table 6. Percentage of Prey Attack Manoeuvres by different types of Gleaner Bird Species in the Tropical Mixed Dry Deciduous Forest, India.

\begin{tabular}{|c|c|c|c|c|c|c|c|c|}
\hline Name of the bird species & $\begin{array}{l}\text { Flower } \\
\text { Gleaner }\end{array}$ & $\begin{array}{l}\text { Fruit } \\
\text { Gleaner }\end{array}$ & $\begin{array}{l}\text { Ground } \\
\text { Gleaner }\end{array}$ & $\begin{array}{l}\text { Leaf } \\
\text { Gleaner }\end{array}$ & $\begin{array}{l}\text { Litter } \\
\text { Gleaner }\end{array}$ & $\begin{array}{l}\text { Trunk } \\
\text { Gleaner }\end{array}$ & $\begin{array}{l}\text { Stem } \\
\text { Gleaner }\end{array}$ & $\begin{array}{l}\text { Twig } \\
\text { Gleaner }\end{array}$ \\
\hline LARGE-BILLED LEAF WARBLER & 0 & 0 & 0 & 90 & 0 & 0 & 0 & 10 \\
\hline BLYTH'S REED WARBLER & 0 & 0 & 0 & 56 & 0 & 0 & 0 & 44 \\
\hline STREAK-THROATED WOODPECKER & 0 & 0 & 0 & 0 & 0 & 65 & 25 & 10 \\
\hline COMMON IORA & 0 & 0 & 0 & 28 & 0 & 0 & 0 & 72 \\
\hline GREENISH WARBLER & 0 & 0 & 0 & 27 & 0 & 0 & 9 & 64 \\
\hline COMMON HOOPOE & 0 & 0 & 46 & 0 & 46 & 4 & 0 & 4 \\
\hline GREY JUNGLEFOWL & 0 & 0 & 74 & 3 & 22 & 0 & 0 & 0 \\
\hline TAWNY-BELLIED BABBLER & 0 & 1 & 0 & 14 & 0 & 0 & 0 & 85 \\
\hline BLACK-HOODED ORIOLE & 0 & 3 & 0 & 0 & 0 & 9 & 50 & 38 \\
\hline COMMON TAILORBIRD & 0 & 5 & 3 & 35 & 0 & 0 & 0 & 58 \\
\hline BLUE-FACED MALKOHA & 0 & 23 & 7 & 10 & 16 & 0 & 8 & 36 \\
\hline BLACK BULBUL & 0 & 32 & 0 & 0 & 0 & 0 & 2 & 66 \\
\hline INDIAN PEAFOWL & 0 & 33 & 54 & 4 & 8 & 0 & 0 & 0 \\
\hline RED-VENTED BULBUL & 0 & 71 & 1 & 4 & 0 & 0 & 12 & 12 \\
\hline BROWN-HEADED BARBET & 0 & 87 & 0 & 6 & 0 & 0 & 6 & 0 \\
\hline YELLOW-BILLED BABBLER & 1 & 0 & 93 & 1 & 4 & 0 & 0 & 2 \\
\hline WHITE-BROWED BULBUL & 1 & 81 & 2 & 1 & 0 & 0 & 0 & 16 \\
\hline MALABAR PARAKEET & 2 & 98 & 0 & 0 & 0 & 0 & 0 & 0 \\
\hline BLOSSOM-HEADED PARAKEET & 11 & 89 & 0 & 0 & 0 & 0 & 0 & 0 \\
\hline PLAIN FLOWERPECKER & 16 & 0 & 0 & 45 & 0 & 0 & 0 & 39 \\
\hline JUNGLE MYNA & 54 & 6 & 40 & 0 & 0 & 0 & 0 & 0 \\
\hline PALE-BILLED FLOWERPECKER & 67 & 0 & 0 & 14 & 0 & 0 & 0 & 19 \\
\hline PURPLE SUNBIRD & 74 & 0 & 0 & 16 & 0 & 0 & 7 & 4 \\
\hline PURPLE-RUMPED SUNBIRD & 88 & 0 & 0 & 5 & 0 & 0 & 0 & 7 \\
\hline LOTEN'S SUNBIRD & 93 & 1 & 0 & 0 & 0 & 0 & 0 & 6 \\
\hline VERNAL HANGING PARROT & 100 & 0 & 0 & 0 & 0 & 0 & 0 & 0 \\
\hline
\end{tabular}


Leaf Gleaning: Large-billed Leaf Warbler, Blyth's Reed Warbler and Plain Flowerpecker used this method with twig and flower gleaning.

Trunk Gleaning: Streak-throated Woodpecker alone used this type of feeding along with gleaning on stem and twig.

Stem Gleaning: Black-hooded Oriole was the only bird species, which used this method. This species also used fruit, trunk and twig as substrate for collecting food.

Twig Gleaning: Common Iora, Greenish Warbler, Tawnybellied Babbler, Black Bulbul, Common Tailorbird and Blue-faced Malkoha were recognized as twig gleaners (Table 6).

\subsection{Position in the Canopy}

Majority of the canopy layers used for foraging of bird species were edge edge (23\%) followed by ground $(18 \%)$ and middle lower $(17 \%)$. Five major canopy layers out of 10 categories were distinctly used by 36 bird species in the tropical mixed dry deciduous forest, India. The major canopy positions foraged were Edge edge, center middle, center edge, middle edge and the birds used ground or air also (Table 7).

\subsubsection{Ground/Air (Under/over Canopy)}

Bird species such as Grey Junglefowl, Red-rumped Swallow, Yellow-billed Babbler, Common Hoopoe, Jungle Babbler, Indian Peafowl, Green Bee-eater, Jungle Myna, Chestnut-headed Bee-Eater and Blue-faced Malkoha occupied this for its prey. Grey Junglefowl and Red-rumped Swallow depend only on these strata and the other bird species extends overlap with other layers in the canopy (Table 7).

\subsubsection{Center Center (Lower Canopy)}

Bird species perched on the middle main axis of the plant canopy were Streak-throated Woodpecker, Black-hooded Oriole and Tawny-bellied Babbler. They also feed on the edge edge and middle middle canopy. No species was restricted to any particular layer alone.

\subsubsection{Middle Edge (Middle Canopy)}

Bird species feeding on the upper canopy was Whitebellied Drongo which feeds on the upper and middle canopy. Center edge (upper canopy): Birds perched for preying over the upper canopy was Common Tailorbird. Edge edge (upper canopy): Twenty-one bird species (Table 7) were feeding on the upper canopy of the plant. Asian Paradise flycatcher and Common Tailorbird exploited food from other canopies too.

\subsection{Specialists}

Among the four dimensions, number of specialists $\left(\mathrm{J}^{\prime}=0\right)$ was more in the substrates (2) method (2) and canopy (1) followed by height (Table 8). Grey Jungle Fowl, Vernal Hanging Parrot and Red-rumped Swallow are specialists as their $\mathrm{J}$ ' values were zero. On the contrary, generalists were Blue-faced Malkoha, Common Tailorbird and Chestnutheaded Bee-eater (Table 8).

\subsection{Niche Overlap}

Niche overlap was calculated with foraging height (12 categories), foraging manoeuvre (20 categories), canopy (10 categories) and foraging substrate (7 categories). Among the foraging dimensions the higest mean niche overlap among the species was found in method (White-browed Bulbul) followed by canopy, height and the lowest in foraging method (Redrumped Swallow).

Height: Blue-faced Malkoha and Purple-rumped Sunbird had the highest mean niche overlap (0.75) while the lowest (0.36) was found in Yellow-billed Babbler (Table 9). Method: The mean niche overlap in feeding method was highest in the White-browed Bulbul (0.83) and lowest (0.14) in the Redrumped Swallow (Table 9). Canopy: The highest mean niche overlap was found in Brown-headed Barbet (0.82) and lowest was in Yellow-billed Babbler (0.42). Substrate: The highest mean niche overlap was in Common Tailorbird (0.63) and the lowest was in Yellow-billed Babbler (Table 9).

Table 7. Percentage of Ten Foraging Canopy Layers preferred by different bird species in the Tropical Mixed Dry Deciduous Forest, India.

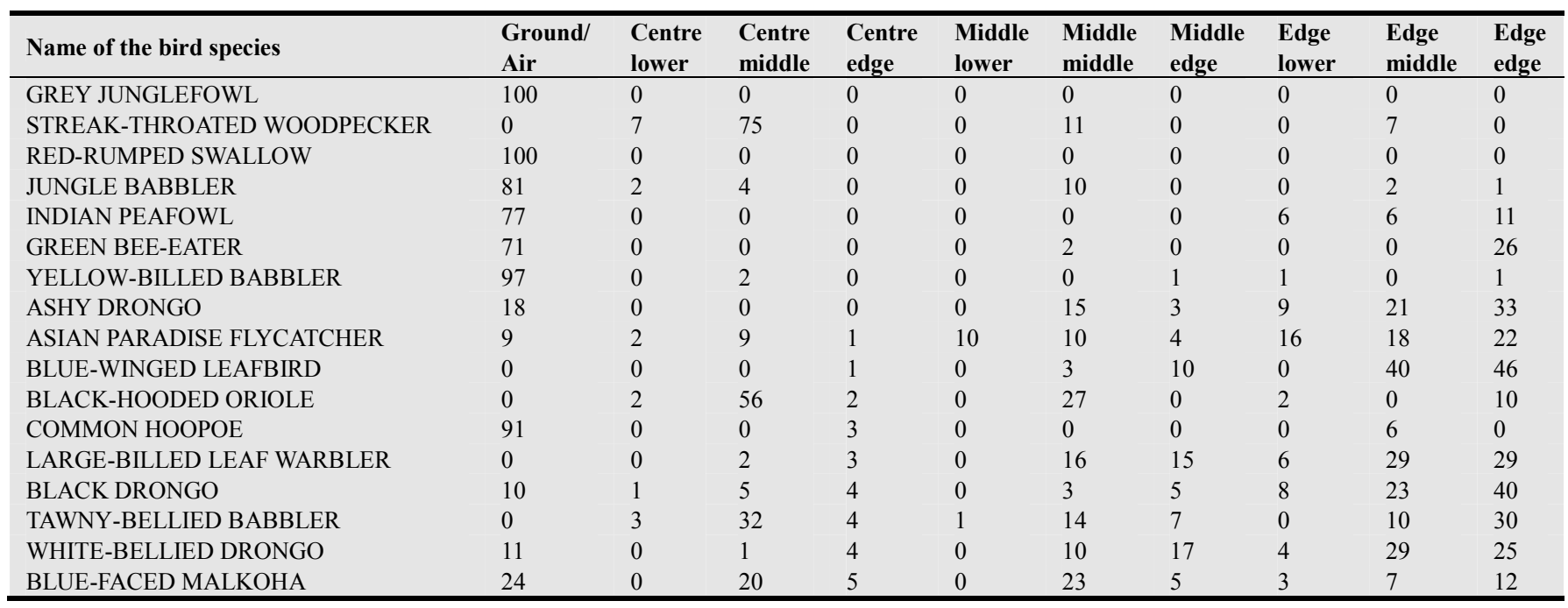




\begin{tabular}{|c|c|c|c|c|c|c|c|c|c|c|}
\hline Name of the bird species & $\begin{array}{l}\text { Ground/ } \\
\text { Air }\end{array}$ & $\begin{array}{l}\text { Centre } \\
\text { lower }\end{array}$ & $\begin{array}{l}\text { Centre } \\
\text { middle }\end{array}$ & $\begin{array}{l}\text { Centre } \\
\text { edge }\end{array}$ & $\begin{array}{l}\text { Middle } \\
\text { lower }\end{array}$ & $\begin{array}{l}\text { Middle } \\
\text { middle }\end{array}$ & $\begin{array}{l}\begin{array}{l}\text { Middle } \\
\text { edge }\end{array} \\
\end{array}$ & $\begin{array}{l}\text { Edge } \\
\text { lower }\end{array}$ & $\begin{array}{l}\text { Edge } \\
\text { middle }\end{array}$ & $\begin{array}{l}\text { Edge } \\
\text { edge }\end{array}$ \\
\hline JUNGLE MYNA & 39 & 0 & 0 & 7 & 1 & 8 & 11 & 0 & 28 & 6 \\
\hline BLYTH'S REED WARBLER & 0 & 0 & 5 & 7 & 2 & 16 & 16 & 5 & 17 & 31 \\
\hline GREENISH WARBLER & 0 & 0 & 8 & 8 & 0 & 17 & 10 & 3 & 31 & 23 \\
\hline PALE-BILLED FLOWERPECKER & 0 & 0 & 2 & 10 & 0 & 9 & 21 & 3 & 11 & 45 \\
\hline RED-WHISKERED BULBUL & 1 & 0 & 7 & 11 & 1 & 2 & 19 & 6 & 13 & 39 \\
\hline BLOSSOM-HEADED PARAKEET & 0 & 0 & 0 & 12 & 0 & 0 & 21 & 0 & 0 & 67 \\
\hline COMMON IORA & 0 & 0 & 4 & 13 & 1 & 18 & 10 & 3 & 20 & 31 \\
\hline PLAIN FLOWERPECKER & 0 & 0 & 4 & 14 & 0 & 7 & 6 & 1 & 19 & 49 \\
\hline WHITE-BROWED BULBUL & 2 & 0 & 5 & 17 & 0 & 10 & 8 & 1 & 16 & 40 \\
\hline MALABAR PARAKEET & 0 & 0 & 4 & 18 & 0 & 0 & 9 & 0 & 9 & 59 \\
\hline LOTEN'S SUNBIRD & 0 & 0 & 1 & 18 & 0 & 4 & 11 & 0 & 10 & 56 \\
\hline PURPLE SUNBIRD & 0 & 0 & 5 & 18 & 2 & 11 & 12 & 4 & 23 & 26 \\
\hline BROWN-HEADED BARBET & 0 & 0 & 3 & 19 & 0 & 10 & 16 & 3 & 13 & 35 \\
\hline PURPLE-RUMPED SUNBIRD & 0 & 0 & 1 & 22 & 1 & 2 & 11 & 4 & 11 & 49 \\
\hline COMMON TAILORBIRD & 3 & 5 & 15 & 23 & 5 & 5 & 8 & 8 & 13 & 18 \\
\hline CHESTNUT-HEADED BEE-EATER & 29 & 0 & 0 & 25 & 0 & 0 & 11 & 5 & 25 & 5 \\
\hline RED-VENTED BULBUL & 2 & 0 & 4 & 26 & 0 & 1 & 10 & 1 & 9 & 47 \\
\hline VERNAL HANGING PARROT & 0 & 0 & 2 & 26 & 0 & 0 & 32 & 2 & 4 & 34 \\
\hline BLACK BULBUL & 0 & 0 & 0 & 35 & 0 & 19 & 9 & 1 & 0 & 35 \\
\hline Total & 765 & 22 & 276 & 356 & 712 & 283 & 318 & 105 & 470 & 981 \\
\hline Total in \% & 18 & 1 & 6 & 8 & 17 & 7 & 7 & 2 & 11 & 23 \\
\hline
\end{tabular}

Table 8. Extent of specialization $\left(J^{\prime}\right)$ by different bird species in foraging substrate, foraging canopy, foraging method and foraging height in the tropical mixed dry deciduous forest, India (J'values range from 001 and specialization increases as $J$ 'decreases; Specialists are indicated in bold numbers).

\begin{tabular}{|c|c|c|c|c|}
\hline \multirow{2}{*}{ Name of the bird species } & Foraging Substrate & Foraging Canopy & Foraging Method & Foraging Height \\
\hline & $\mathbf{J}^{\prime}$ & $\mathbf{J}^{\prime}$ & $\mathbf{J}^{\prime}$ & $\mathbf{J}^{\prime}$ \\
\hline GREY JUNGLEFOWL & 0.09 & 0.00 & 0.52 & 0.00 \\
\hline INDIAN PEAFOWL & 0.45 & 0.37 & 0.89 & 0.56 \\
\hline BLOSSOM-HEADED PARAKEET & 0.17 & 0.40 & 0.15 & 0.11 \\
\hline MALABAR PARAKEET & 0.06 & 0.58 & 0.05 & 0.44 \\
\hline VERNAL HANGING PARROT & 0.00 & 0.64 & 0.00 & 0.33 \\
\hline BLUE-FACED MALKOHA & 1.00 & 0.88 & 1.00 & 0.85 \\
\hline CHESTNUT-HEADED BEE-EATER & 0.26 & 0.75 & 0.65 & 1.00 \\
\hline GREEN BEE-EATER & 0.35 & 0.32 & 0.41 & 0.26 \\
\hline COMMON HOOPOE & 0.24 & 0.17 & 0.92 & 0.70 \\
\hline STREAK-THROATED WOODPECKER & 0.18 & 0.39 & 0.22 & 0.11 \\
\hline REDRUMPED SWALLOW & 0.00 & 0.00 & 0.00 & 0.22 \\
\hline BLACK-HOODED ORIOLE & 0.49 & 0.56 & 0.78 & 0.52 \\
\hline BLACK DRONGO & 0.05 & 0.82 & 0.72 & 0.89 \\
\hline ASHY DRONGO & 0.37 & 0.76 & 0.68 & 0.71 \\
\hline WHITE-BELLIED DRONGO & 0.06 & 0.83 & 0.74 & 0.78 \\
\hline JUNGLE MYNA & 0.66 & 0.75 & 0.57 & 0.56 \\
\hline COMMON IORA & 0.41 & 0.84 & 0.37 & 0.33 \\
\hline BLUE-WINGED LEAFBIRD & 0.78 & 0.52 & 0.68 & 0.52 \\
\hline RED-WHISKERED BULBUL & 0.55 & 0.82 & 0.49 & 0.81 \\
\hline RED-VENTED BULBUL & 0.64 & 0.68 & 0.58 & 0.67 \\
\hline WHITE-BROWED BULBUL & 0.51 & 0.79 & 0.45 & 0.33 \\
\hline BLACK BULBUL & 0.63 & 0.63 & 0.56 & 0.54 \\
\hline TAWNY-BELLIED BABBLER & 0.31 & 0.80 & 0.28 & 0.26 \\
\hline JUNGLE BABBLER & 0.39 & 0.35 & 0.64 & 0.26 \\
\hline YELLOW-BILLED BABBLER & 0.09 & 0.08 & 0.17 & 0.15 \\
\hline ASIAN PARADISE FLYCATCHER & 0.25 & 0.98 & 0.62 & 0.52 \\
\hline COMMON TAILORBIRD & 0.63 & 1.00 & 0.56 & 0.33 \\
\hline BLYTH'S REED WARBLER & 0.47 & 0.21 & 0.41 & 0.54 \\
\hline LARGE-BILLED LEAF WARBLER & 0.22 & 0.78 & 0.20 & 0.56 \\
\hline GREENISH WARBLER & 0.59 & 0.82 & 0.51 & 0.56 \\
\hline BROWN-HEADED BARBET & 0.32 & 0.80 & 0.28 & 0.85 \\
\hline PALE-BILLED FLOWERPECKER & 0.61 & 0.73 & 0.54 & 0.70 \\
\hline PLAIN FLOWERPECKER & 0.67 & 0.70 & 0.60 & 0.67 \\
\hline PURPLE-RUMPED SUNBIRD & 0.33 & 0.68 & 0.28 & 0.33 \\
\hline LOTEN'S SUNBIRD & 0.19 & 0.60 & 0.16 & 0.63 \\
\hline PURPLE SUNBIRD & 0.55 & 0.86 & 0.49 & 0.81 \\
\hline Number of specialists & 2 & 2 & 2 & 1 \\
\hline Number of generalist & 1 & 1 & 1 & 1 \\
\hline
\end{tabular}


Table 9. Mean niche overlap for different bird species in foraging height, foraging substrate, foraging canopy and foraging method in the tropical mixed dry deciduous forest, India (niche overlap ranges from 001 and high niche overlap is indicated in bold numbers).

\begin{tabular}{|c|c|c|c|c|c|}
\hline \multirow{2}{*}{ Name of the Bird species } & \multicolumn{5}{|l|}{ Niche overlap } \\
\hline & Foraging Height & Foraging Substrate & Foraging Canopy & Foraging Method & All dimensions \\
\hline ASHY DRONGO & 0.68 & 0.54 & 0.80 & 0.56 & 0.67 \\
\hline BLACK BULBUL & 0.59 & 0.58 & 0.71 & 0.82 & 0.63 \\
\hline BLACK DRONGO & 0.66 & 0.43 & 0.81 & 0.26 & 0.63 \\
\hline BLACK-HOODED ORIOLE & 0.69 & 0.54 & 0.61 & 0.77 & 0.61 \\
\hline BLOSSOM-HEADED PARAKEET & 0.54 & 0.51 & 0.73 & 0.82 & 0.59 \\
\hline BLYTH'S REED WARBLER & 0.66 & 0.51 & 0.79 & 0.82 & 0.65 \\
\hline MALABAR PARAKEET & 0.71 & 0.36 & 0.72 & 0.82 & 0.59 \\
\hline BLUE-FACED MALKOHA & 0.75 & 0.61 & 0.76 & 0.82 & 0.70 \\
\hline GREY JUNGLEFOWL & 0.38 & 0.42 & 0.43 & 0.82 & 0.41 \\
\hline BLUE-WINGED LEAFBIRD & 0.66 & 0.57 & 0.71 & 0.81 & 0.64 \\
\hline COMMON HOOPOE & 0.53 & 0.52 & 0.58 & 0.75 & 0.54 \\
\hline COMMON IORA & 0.73 & 0.45 & 0.78 & 0.82 & 0.65 \\
\hline JUNGLE BABBLER & 0.51 & 0.42 & 0.53 & 0.82 & 0.48 \\
\hline JUNGLE MYNA & 0.61 & 0.50 & 0.73 & 0.82 & 0.61 \\
\hline LARGE-BILLED LEAF WARBLER & 0.71 & 0.44 & 0.76 & 0.82 & 0.63 \\
\hline BROWN-HEADED BARBET & 0.73 & 0.55 & 0.82 & 0.82 & 0.69 \\
\hline VERNAL HANGING PARROT & 0.49 & 0.46 & 0.74 & 0.82 & 0.56 \\
\hline LOTEN'S SUNBIRD & 0.70 & 0.46 & 0.76 & 0.82 & 0.64 \\
\hline STREAK-THROATED WOODPECKER & 0.70 & 0.51 & 0.57 & 0.74 & 0.59 \\
\hline PLAIN FLOWERPECKER & 0.70 & 0.59 & 0.79 & 0.82 & 0.69 \\
\hline INDIAN PEAFOWL & 0.56 & 0.55 & 0.65 & 0.73 & 0.59 \\
\hline ASIAN PARADISE FLYCATHER & 0.63 & 0.43 & 0.77 & 0.26 & 0.60 \\
\hline GREENISH WARBLER & 0.69 & 0.51 & 0.77 & 0.82 & 0.65 \\
\hline PURPLE-RUMPED SUNBIRD & 0.75 & 0.42 & 0.74 & 0.82 & 0.63 \\
\hline PURPLE SUNBIRD & 0.72 & 0.54 & 0.80 & 0.82 & 0.68 \\
\hline TAWNY-BELLIED BABBLER & 0.49 & 0.51 & 0.73 & 0.82 & 0.57 \\
\hline REDRUMPED SWALLOW & 0.53 & 0.36 & 0.49 & 0.14 & 0.46 \\
\hline RED-VENTED BULBUL & 0.68 & 0.52 & 0.77 & 0.82 & 0.65 \\
\hline RED-WHISKERED BULBUL & 0.74 & 0.54 & 0.80 & 0.82 & 0.69 \\
\hline GREEN BEE0EATER & 0.64 & 0.47 & 0.66 & 0.26 & 0.59 \\
\hline COMMON TAILORBIRD & 0.68 & 0.63 & 0.78 & 0.82 & 0.69 \\
\hline PALE-BILLED FLOWERPECKER & 0.74 & 0.54 & 0.78 & 0.82 & 0.68 \\
\hline WHITE-BROWED BULBUL & 0.66 & 0.47 & 0.80 & 0.83 & 0.64 \\
\hline WHITE-BELLIED DRONGO & 0.69 & 0.40 & 0.80 & 0.27 & 0.63 \\
\hline YELLOW-BILLED BABBLER & 0.36 & 0.35 & 0.42 & 0.82 & 0.38 \\
\hline CHESTNUT-HEADED BEE-EATER & 0.71 & 0.47 & 0.73 & 0.26 & 0.63 \\
\hline
\end{tabular}

All dimensions: All the dimensions together when combined, Yellow-billed Babbler showed the lowest overlap (0.38) and Blue-faced Malkoha (0.70) showed the highest overlap among the 36 species (Table 9).

\subsection{Foraging Guilds}

Species were separated into a number of distinct groups whose members exploit food resources from similar substrates or height using similar methods and thereby considered as guilds. The guild formed in the tropical mixed dry deciduous forest, India based on the use of substrates, methods, canopy and height, their relationships among the 36 bird species are summarized in the cluster diagram (Figure 2). Two distinct major guilds (gleaner and sallier) were arbitrarily recognized from the cluster diagram (Figure 2). The gleaner was further consisted of three distinct guilds based on the substrates of gleaning, namely 1. Fruit, 2. Flower, 3. Ground and 4. Stem (trunk and twigs).

Guild I consisted of birds that glean their prey on fruit (Frugivore). Guild II consisted of birds that glean their food from the flower (Nectarivore). The guild III consisted of birds that largely obtained their food mainly insects or other invertebrates from all strata (ground, plant and air) (Figure 2). Within this guild, two major groups were obvious such as purely insectivore and omnivore. This was bifurcated again into five groups based on the substrates: ground, twigs and leaf, main trunk and air. 


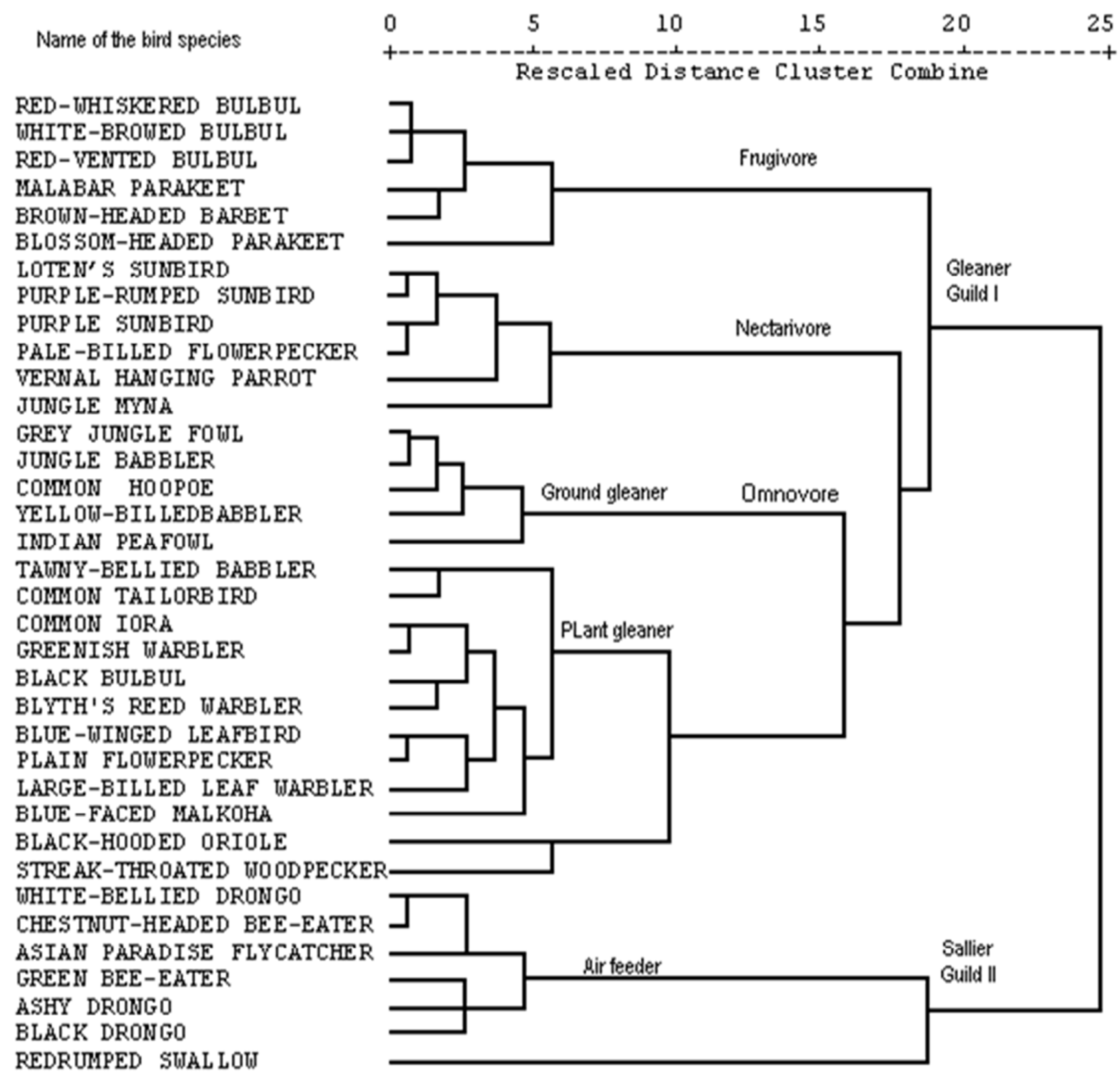

Figure 2. Dendrogram showing interspecific relationships of 36 bird species based on multivariate analyses of foraging method, substrate and height use in the tropical mixed dry deciduous forest, India.

Based on the observational data, birds foraged in similar ways or exploited the same resources for foods were grouped in a schematic representation (Figure 3). The schematic portrayal of the groupings relies on the foraging behavior, foraging height, canopy and foraging substrate differences to associate species. Of the 36 species, major group of birds was of insectivores, which comprised of 24 bird species followed by nectarivores such as Vernal Hanging Parrot, Loten's Sunbird, Purple-rumped Sunbird, Pale-billed Flowerpecker and Jungle Myna. Frugivore guild comprised of (fruit, flower, insect and grain feeder) Red-whiskered Bulbul, Redvented Bulbul, White-browed Bulbul, Malabar Parakeet, Brown-headed Barbet and Blossom-headed Parakeet. Insectivores largely obtain their food from plants or from air by sally (Red-rumped Swallow). Among the plant forms, the number of species, which obtained their food from twig and leaf were more than that depending on other substrates such as main trunk and secondary branches. Six bird species sallying from four different positions in the canopy were distinguished as insectivore's viz. Chestnut-headed Bee-eater, White-bellied Drongo, Ashy Drongo, Paradise Flycatcher and Green Bee-eater (Figure 3). Other insectivores guild, feeding from plants were Streak-throated Woodpecker, Black-hooded Oriole, Tawny-bellied Babbler, Common Tailorbird, Common Iora, Greenish Warbler, Blyth's Reed Warbler, Blue-winged Leafbird, Plain Flowerpecker, Large-billed Leaf Warbler and Blue-faced Malkoha. 


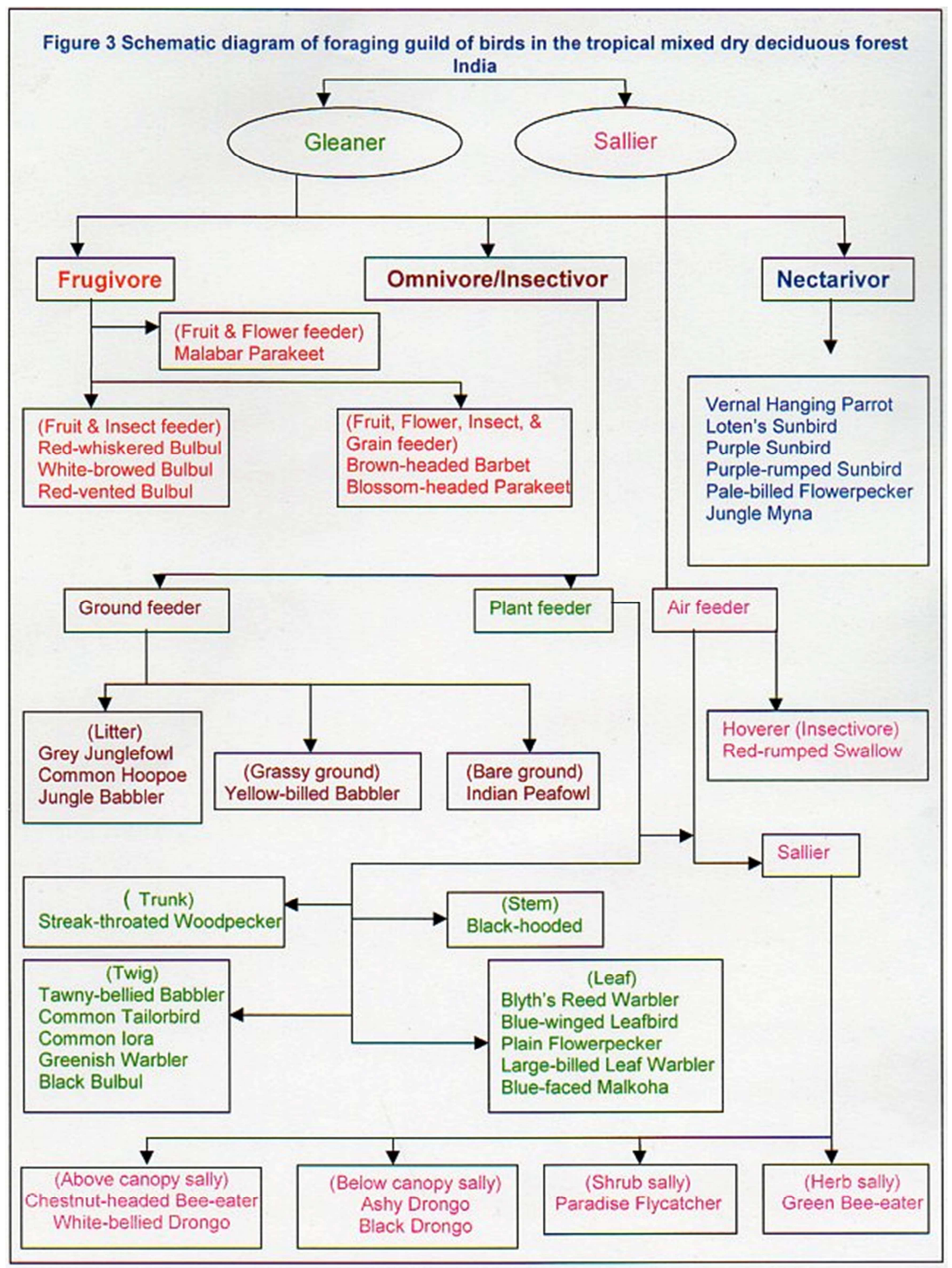

Figure 3. Schematic diagram of foraging guild of birds in the trophical mixed dry desiduous forest india.

\subsection{Plant Community in the Tropical Mixed Dry Deciduous} Forest

Vegetation profile of mixed dry deciduous forest consisted mostly of tree species of 2-6m height (Figure 4) and the upper stratum was thinned out with a few tall trees such as Ficus sp., Tamarindus indica, Acacia polyacantha, Albizia amara, Canthium dicoccum, Celtis philippensis and Commiphora caudata. Shrubs formed the lower stratum at 0$2 \mathrm{~m}$. and it occupied a predominant place from ground to $1 \mathrm{~m}$ height in mixed dry deciduous forest. Moreover the number of shrub species are higher (45) than the tree species (27). Higher foliage profile layers harbour more bird species [19] was true in this habitat as studied by [20]. 


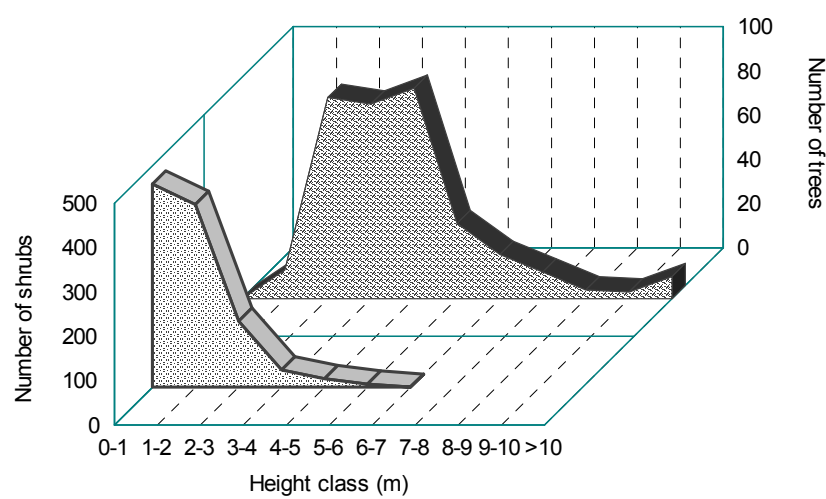

Figure 4. Vegetation profile of mixed dry deciduous forest.

\section{Discussion}

Tree layers found to be a distinctive foraging environment for birds in the tropical mixed dry deciduous forest, India followed by shrubs/short tree layers due to the availability of high foliage layer in the trees and more foliage overlap between short trees and shrubs. Sucessful foraging by avian predators is influenced largely by prey availabilty, which encompasses not only the density of prey but also its vulnerabilty to capture [21]. An intersting observation was this forest comprised of two guilds namely gleaners and salliers. Feeding methods are more specialised in each species. Species generalised in feeding tend to vary in feeding technique, substrate choice, canopy and height when the type of food varies. Yet another intersting observation was large scale utilization of layers at different height such as $0.1-2 \mathrm{~m}$ and $3-6 \mathrm{~m}$. This might perhaps be due to the foliages of majority of the trees in the tropical mixed dry deciduous forest of India are spread between 3-6m height which formed the upper stratum and shrubs of $0-2 \mathrm{~m}$ height formed the lower stratum and that gives more opportunity to birds for exploitation. Moreover the number of shrub species are higher than the tree species. The availability of various plant forms such as shrubs, short trees and trees in these habitats not only increases the vertical and horizontal foliage layering and complexity, but also provides many supporting substrates. So majority of birds in this habitat used these strata for foraging. Foraging birds require a large number of small preys to maintain resting metabolic rates [22]. Information on the foraging height, attack maneuvers; substrate and foliage density was collected independently for each foraging bird [23].

Three major substrates namely ground, plant and air were recognized. Of which, more bird species fell under the plant guild because plant offers a great variety of microhabitats (trunk, branches, twigs, foliage, flower and fruit) to find their suitable and favourable food. Foliage and twigs were utilized by more number of birds because branches with leaves offer a great variety of places to find food along with concealment. Moreover most of the trees in this habitat withered their dryleaves and emerging of new leaves tookplace during winter, thus increasing the opportunity of searching and finding their prey or vicinity of the prey becomes more. In total, bird species used 12 methods to obtain food from the tropical mixed dry deciduous forest of India. Searching patterns are largely a function of the morphological and perceptual traits of each species, which allow the birds to move through the foliage to locate, detect and capture prey in specific ways. Similar study was reported in thorn forest [5] of Mudumalai Wildlife Sanctuary, India. Information on the foraging height, attack maneuvers; substrate and foliage density was collected independently for each foraging bird [23].

The availability of diverse food items may vary between habitats [20] and hence birds that feed on variety of foods (e.g., insects, seed, nectar and fruit) may change their manoeuvre according to the habitat. Moreover, changes in the foraging manoeuvres may be a strategy to avoid competition. Hence it is likely that the combination of factors such as availability of food, habitat structure and interspecific competition are responsible for the variations in the foraging behaviour of birds observed in this forest. Predation of two adult birds was recorded during the study period. Also, predation of fledglings of almost all the breeding birds was observed. Interspecific competition also can alter foraging behavior of Warblers and Babblers [24], [25], [26]. Thus, changes in the foraging manoeuvre may be a strategy to avoid competition. Hence, it is likely that the combination of factors such as availability of food, habitat structure and interspecific competition are responsible for the changes. Foraging behavior and foraging success of the reddish egret were studied by [27] focusing on whether their foraging behavior or success varied with age, color morph, group size and habitat measures.

In this study, closely related species used the same basic foraging method indicating the importance of phylogeny in determining the feeding patterns of birds [28], [4]. Resource partitioning reduces the effect of competition by decreasing the amount of overlap between the competing species [2]. Partitioning of foraging dimensions among birds could occur in this habitat as reported earlier for bird communities of various places and habitats [6], [29], [5], [20] Foraging behavior and foraging success of the reddish egret were studied by [27] focusing on whether their foraging behavior or success varied with age, color morph, group size and habitat measures.

Many species fed from different strata and positions in the canopy overlapping with others where specialists such as the Yellow-billed Babbler fed by only gleaning and that too from ground thus sharing high specialization or preference and thus having very little overlap with other species. When food availability is high they feed on the outer part of tree canopies in this study as found by Diaz et al. [30] in Tits. Birds selected foraging sites with a higher mean prey density than at random sites [31].

Some species of water birds have been found to forage at the interface of open water and vegetation [32], [33], [34].

Bird species evolved with specialization for a particular type of habitat or substrate or prey that resulted in a specialist for a particular habitat. Greenberg [35], [36] investigated 
Warblers' response to different substrate and inferred that the species that had a diverse foraging behavior were conservative in their use of substrates. Thus it can be inferred that niche overlap can be attributed to the availability of food resources, morphology of species and competition as suggested by Alatalo [24], Rolando and Robotti [37], Szekely [38] and Gokula and Vijayan [5]. Successful foraging by avian predators is influenced largely by prey availability, which encompasses not only the density of prey but also its vulnerability to capture [21].

\section{Conclusion}

Foraging data were collected early in the morning during the study period. In total, 36 species were observed from the mixed dry deciduous forest. Various foraging dimensions such as method, substrate, height and position in the canopy were analyzed. Foraging attempts were assigned to 12 height categories, seven substrate categories, 9 positions in the canopy and 20 foraging methods. Thirteen species shared change in the use of substrate while only five species changed the method used. Five bird species were considered as specialists as their J' values were zero. In four dimensions highest mean niche overlap is found in the use of foraging height. There are two major guilds, namely gleaners and salliers and gleaners are grouped into four major guilds. There are four major groupings among the bird species based on the food eaten such as insectivores, nectarivores, frugivores and omnivores. The plant (shrubs and trees) surface provides microhabitats such as foliage, twig, flower, fruit, secondary branches and trunk and the proportion of foliage use at different heights is higher. Specialization of species and their niche overlap with others are analysed. Foraging method is specialized being constrained by morphology in many species while substrates and strata are used opportunistically depending on the environment.

\section{References}

[1] N. B. Davies, "Ecological questions about territorial behaviour. In Behavioural ecology- an evolutionary approach" (Eds.) J. R. Krebs and N. B. Davies. Blackwell scientific publications. Oxford, London Edinburgh and Melbourne. 1978, pp. 317-350.

[2] J. A. Wiens, "Ecology of bird communities". Cambridge University Press, Cambridge, 1989 Vol. 1 \& 2.

[3] F. H. J Crome, Foraging ecology of an assemblage of birds in lowland rainforest in Northern Queensland. Aust. J. Ecol. 3: 1978, pp. 195-212.

[4] R. T. Holmes, R. E. Jr. Bonney and S. W Dalala, "Guild structure of the Hubbard Brook bird community: a multivariate approach" Ecology. 60: 1979, pp. 512-520.

[5] V. Gokula and L. Vijayan, "Foraging patterns of birds in the thorn forest of Mudumalai Wildlife Sanctuary, Southern India” J. South Asian Nat. Hist. 5 (2): 2001, pp. 143-152.

[6] H. F. Recher, R. T. Holmes, M. Schulz, J. Shields and R.
Kavanagh, "Foraging patterns of breeding birds in eucalyptus forest and woodland of Southern Australia". Aust. J. Ecol. 10: 1985, pp. 399-419.

[7] R. Mac Nally, "Habitat specific guild structure of forest birds in southeastern Australia: a regional scales perspective", $J$. Anim. Ecol. 63: 1994, pp. 988-1001.

[8] H. G. Champion and S. K. Seth, "A revised survey of the forest types of India", Government of India publications". New Delhi, India, 1968.

[9] C. J. Bibby, N. D Burgess and D. A. Hill, "Bird census techniques. British Trust for Ornithology and the Royal Society for the Protection of Birds" Academic Press, London. 1993, pp. 66-84.

[10] G. P. Bell, G. A. Bartholomew and K. A. Nagy, "The roles of energetics, water economy, foraging behavior, and geothermal refugia in the distribution of the bat, Macrotus californicus". J. Comp. Psych. B. 156: 1986, pp. 441-450.

[11] J. V. Ramsen and S. K. Robinson, - A classification scheme for foraging behaviour of birds in terrestrial habitats. Studies in Avian Biology 123: 1990, 144-160.

[12] R. C. Mac Nally, "On characterizing foraging versatalization illustrated by using birds" Oikos. 69: 1994, pp. 95-106.

[13] M. L. Morrison, "Influence of sample size and sampling design on analysis of avian foraging behavior" Condor. 86: 1984, 146-150.

[14] C. E. Shannon and W. Weaver, "The mathematical theory of communication. University of Illinois Press", Urbana, 1949.

[15] H. S. Horn, - The measurement of 'overlap' in comparative ecological studies. Am. Nat. 100: 1966, pp. 419-424.

[16] L. Legendre and P. Legendre, - Numerical ecology. Developments in environmental modelling, Elsevier Sci. Publ. Co., Amsterdem, 1983.

[17] F. L Rohlf., "Numerical Taxonomy and Multivariate Analysis System". Exeter publishing, Setauket, New York 1987.

[18] M. J. Norusis, -“SPSS Inc. SPSS release 6.0 for Unisys 6000”, Chicago, Illinois, USA, 1994.

[19] R. H. Mac Arthur and J. W. MacArthur, - On bird species diversity. Ecology. 42 (3): 1961, pp. 594-598.

[20] T. Nirmala, L. Vijayan, "Breeding behaviour of the Indian Robin Saxicoloides fulicata in the Anaikatty hills, Coimbatore", pp. 43-46. In: Proceedings of 28th ESI Conference, (2003), pp. 7-8.

[21] M. Samantha, Lantz, E. Dale, Gawlie, I. Marce and Cook, "The effect of water depth and submerged Aquatic vegetation on the success of wading birds Condor 112 (3): 2010, pp. 460-469.

[22] T. Piersma, "Energetic bottles and other de-sing constrains in avian annual cycles" Integrative and comparative Biology, 42: 2002, pp. 51-67.

[23] S. Mohammad, Mansor and A. Shahrul Mohamed Sah, "Foraging Patterns reveal niche separation in tropical insectivorous birds", Acta Ornithologic Vol. 47, 2012.

[24] R. V. Alatalo, "Interspecific competition in tits Parus spp. and the Goldcrest Regulus regulus: foraging shifts in multispecific flocks" Oikos. 37: 1981, pp. 335-344. 
[25] L. Vijayan, "Comparative biology of Drongos (Family: Dicruridae, Class: Aves) with special reference to ecological Isolation" Ph. D. Thesis, Bombay University, Bombay, 1984.

[26] J. H. Carothers, -"Behavioral and ecological correlates of interference competition among some Hawaiian drepanidinae" Auk. 103: 1986, pp. 564-574.

[27] M. Elizabeth Baters and Bart M. Ballard "Factors influencing behavior and success of foraging Reddish Egrets (Egretta rufescents)", water birds 37 (2): 2014, pp. 191-202.

[28] S. K. Robinson and R. T. Holmes, -"Foraging behaviour of forest birds: the relationships among search tactics, diet, and habitat structure", Ecology. 63: 1982, pp. 1918-1931.

[29] A. G. Wheeler and M. C. Calver, "Resource partitioning in an Island community of insectivores birds during winter" Emu. 96: 1996, pp. 23-31.

[30] M. Diaz, J. C Illera. and J. C. Atienza, "Food resource matching by foraging tits parus spp. during spring - summer in a mediterranean mixed forest; evidence for an ideal free distribution" Ibis. 140: 1998, pp. 654-660.

[31] L. Racheal, Pierce and E. Dale Gawlik "Wadding birds foraging habitats selection in the florida Everglader" Water birds 33 (4): 2010, pp. 494-503.
[32] R. J. Saffron, M. A. Colwell, C. R. Isola and O. E. Taft, "Foraging site selection by nonbreeding White-faced Ibis" Condor 102: 2000, pp. 221-225.

[33] R. E. Bennetts, P. C. Darby and L. B. Karunaratne, "Foraging patch selection by snail kites in response to vegetation structure and prey abundance and availability" Water birds 29: 2006, pp. 88-94.

[34] E. D. Stolen, J. A. Collazo and H. F Percivan, "Vegetation effects on fish distribution in impounded salt marshes" South Eastern Naturalist 8: 2010, pp. 503-514.

[35] R. Greenberg, - The Winter exploitation systems of Baybreasted and Chestnut-sided warblers in Panama. University of California. Publ. Zool. 116: 1984a, pp. 1-107.

[36] R. Greenberg, "Neophobia in the foraging site selection of a neotropical migrant bird: an experimental study. Proc. Nat. Acad. Sci. 81: 1984 b pp. 3778-3780.

[37] A. Rolando and C. A Robotti., "Foraging niches of tits and associated species in north-western Italy" Boll. Zool. 52: 1985, pp. 281-297.

[38] T. Szekely, "Foraging structure of the foliage-gleaning and bark-foraging guild in winter and spring. Proc. Fifth Nordic Ornithological Congress: 1985, pp. 140-146. 